OPEN ACCESS

Edited by:

Alain De Bruin,

Utrecht University, Netherlands

Reviewed by:

Bin Zhou,

Shanghai Institute of Biochemistry and Cell Biology (CAS), China Marcos Malumbres, Spanish National Cancer Research

Center, Spain

*Correspondence:

Deqiang $\mathrm{Li}$

dqli@som.umaryland.edu

${ }^{\dagger}$ These authors have contributed equally to this work

Specialty section:

This article was submitted to

Cell Growth and Division,

a section of the journal

Frontiers in Cell and Developmental

Biology

Received: 14 February 2020

Accepted: 29 April 2020

Published: 25 May 2020

Citation:

Jang J, Engleka KA, Liu F, Li L,

Song G, Epstein JA and Li D (2020)

An Engineered Mouse to Identify

Proliferating Cells and Their

Derivatives.

Front. Cell Dev. Biol. 8:388.

doi: 10.3389/fcell.2020.00388

\section{An Engineered Mouse to Identify Proliferating Cells and Their Derivatives}

\author{
Jihyun Jang ${ }^{1 \dagger}$, Kurt A. Engleka ${ }^{2,3+}$, Feiyan Liu ${ }^{2,3}$, Li Li ${ }^{3}$, Guang Song ${ }^{1}$, \\ Jonathan A. Epstein ${ }^{2,3}$ and Deqiang Li* ${ }^{1 *}$
}

${ }^{1}$ Department of Surgery, Center for Vascular and Inflammatory Diseases, University of Maryland School of Medicine, Baltimore, MD, United States, ${ }^{2}$ Department of Cell and Developmental Biology, Perelman School of Medicine, University of Pennsylvania, Philadelphia, PA, United States, ${ }^{3}$ Penn Cardiovascular Institute, Perelman School of Medicine, University of Pennsylvania, Philadelphia, PA, United States

Background: Cell proliferation is a fundamental event during development, disease, and regeneration. Effectively tracking and quantifying proliferating cells and their derivatives is critical for addressing many research questions. Cell cycle expression such as for Ki67, proliferating cell nuclear antigen (PCNA), or aurora kinase B (Aurkb), or measurement of 5-bromo-2' -deoxyuridine (BrdU) or ${ }^{3} \mathrm{H}$-thymidine incorporation have been widely used to assess and quantify cell proliferation. These are powerful tools for detecting actively proliferating cells, but they do not identify cell populations derived from proliferating progenitors over time.

Aims: We developed a new mouse tool for lineage tracing of proliferating cells by targeting the Aurkb allele.

Results: In quiescent cells or cells arrested at G1/S, little or no Aurkb mRNA is detectable. In cycling cells, Aurkb transcripts are detectable at $\mathrm{G} 2$ and become undetectable by telophase. These findings suggest that Aurkb transcription is restricted to proliferating cells and is tightly coupled to cell proliferation. Accordingly, we generated an Aurkb $b^{E R ~ C r e /+}$ mouse by targeting a tamoxifen inducible Cre cassette into the start codon of Aurkb. We find that the Aurkb $E R$ Cre/+ mouse faithfully labels proliferating cells in developing embryos and regenerative adult tissues such as intestine but does not label quiescent cells such as post-mitotic neurons.

Conclusion: The AurkbER Cre/+ mouse faithfully labels proliferating cells and their derivatives in developing embryos and regenerative adult tissues. This new mouse tool provides a novel genetic tracing capability for studying tissue proliferation and regeneration.

Keywords: cell proliferation, aurora kinase B, mouse model, lineage tracing, regeneration, development

\section{INTRODUCTION}

Cell proliferation is a fundamental biological event in all multicellular organisms (Nasmyth, 2001). Identification or quantification of proliferating cells is essential to understanding organogenesis, morphogenesis, tumorigenesis, and regeneration. Replicating cells can be identified based on expression of cell-cycle markers, such as Ki67, proliferating cell nuclear antigen (PCNA), aurora 
kinase B (Aurkb), or the incorporation of thymidine analogs, such as ${ }^{3} \mathrm{H}$-thymidine, 5-bromo-2'-deoxyuridine (BrdU), or 5ethynyl-2'-deoxyuridine (EdU) (Mitchison and Salmon, 2001). Incorporation of 5-iodo-2'-deoxyuridine (IdU) has been used to analyze proliferation in human tissue (Pan et al., 2013). These assays are suitable for detecting actively proliferating or labelretaining cells. However, retrospective lineage tracing is often desired when proliferating cells must be tracked for their growth pattern or quantity under certain biological conditions such as tissue morphogenesis or regeneration.

Genetic engineering in the mouse allows lineage tracing mouse models to track the derivatives of proliferating cells. One model is the mosaic analysis with double markers (MADM) mouse model, which labels dividing cells through interchromosomal recombination (Zong et al., 2005), although its application is limited due to low labeling efficiency. More recently, a Ki67 ${ }^{\mathrm{RESSCreER} /+}$ mouse was generated and used to track proliferating cells in brain or heart (Basak et al., 2018; Kretzschmar et al., 2018). However, Ki67 is expressed throughout the cell cycle including G1, and some non-proliferative cells such as adult cardiomyocytes can poise at G1 for an extended period of time without cell division (Alvarez et al., 2019).

Aurkb, a key component of the chromosomal passenger complex, localizes to the centromeres to ensure precise chromosome segregation during mitosis and to the midbody to assist cytoplasmic separation during cytokinesis (van der Waal et al., 2012). Knockdown or inhibition of Aurkb in vitro inhibits cell proliferation (Yu et al., 2015; Helfrich et al., 2016), while knockout of Aurkb in mice results in mitotic defects in the inner cell mass (Fernandez-Miranda et al., 2011). Increased expression of Aurkb is associated with tumorigenesis and inhibition of Aurkb may be an effective cancer therapeutic target (Tang et al., 2017; Tischer and Gergely, 2019). Aurkb has been widely used to identify mitotic cells using immunofluorescence or immunohistochemical methods with anti-Aurkb antibodies (Vader and Lens, 2008; Liu and Lampson, 2009; van der Waal et al., 2012; Tian et al., 2015; Nakada et al., 2017; Yu et al., 2019).

In order to track cell proliferation retrospectively, we have generated $A u r k b^{E R C r e /+}$ mice by targeting a tamoxifen inducible Cre cassette into the start codon of Aurkb. By characterizing the $A u r k b^{E R C r e}$ allele in vitro and in vivo, we show that $A u r k b b^{E R ~ C r e /+}$ mice faithfully label proliferating cells and their derivatives during development and regeneration.

\section{MATERIALS AND METHODS}

\section{Mice}

Aurkb ${ }^{E R C r e /+}$ mice were generated by homologous recombination in embryonic stem cells targeting a CreErt2-V2A-tdTomato-Frt-PGK-neo-Frt cassette into the start codon of the Aurkb locus. Thus, the insertion of this cassette will lead to the ablation of endogenous Aurkb expression in the target allele. The PGK-Neo cassette was removed by breeding the initial progeny to mice expressing ubiquitous
FlpE recombinase (Rodriguez et al., 2000). Southern blot confirmed the expected homologous recombination and germ line transmission of the targeted allele. The Aurkb ${ }^{E R C r e}$ allele is detected by PCR using the following primers: Forward: 5'-GTGGGCTCTATGGCTTCTGA-3', Reverse (common): 5'-CAAATTCTTGAGGCCCACAC-3'; product size: $501 \mathrm{bp}$. The wild-type allele is detected by using the following primers: Forward: 5'-ATGGACCTAGAGCGGGAGAT- $3^{\prime}$ and Reverse (common); product size: $264 \mathrm{bp}$. The V2A-tdTomato included in the targeting construct potentially provides a means to fluorescently label Aurkb-expressing cells without disrupting Cre-Ert2 function. However, although we were able to detect tdTomato protein expression by immunofluorescence using antibodies on fixed intestinal crypts (Supplementary Figure 1), the spontaneous tdTomato fluorescence was below levels of detection. B6.129 × 1-Gt (ROSA) 26Sor TM1(EYFP) Cos $/+$ (abbreviated as $R 26 R^{\text {eYFP }}$ ) mice were purchased from The Jackson Laboratory (stock number: 006148). All mice were maintained on a mixed genetic background. All animal protocols were approved by the University of Pennsylvania Institutional Animal Care and Use Committee (IACUC \#: 803396) and the University of Maryland Baltimore Institutional Animal Care and Use Committee (IACUC \#: 0118005).

\section{Administration of Tamoxifen and 5-Bromo-2'-Deoxyuridine (BrdU) in vivo}

Tamoxifen (Sigma-Aldrich, St. Louis, MO, United States) $(10 \mathrm{mg} / \mathrm{ml})$ was dissolved in corn oil. Tamoxifen [2 or 100 or $150 \mathrm{mg} / \mathrm{kg}$ body weight (BW)] was given to Aurkb ${ }^{E R ~ C r e /+}$; $R 26 R^{e Y F P /+}$ mice by either intraperitoneal injection or gavage. BrdU (Sigma-Aldrich, St. Louis, MO, United States) $(10 \mathrm{mg} / \mathrm{ml})$ was dissolved in phosphate-buffered saline (PBS) and intraperitoneally delivered to Aurkb ${ }^{E R C r e /+} ; R 26 R^{e Y F P /+}$ mice $(100 \mathrm{mg} / \mathrm{kg} \mathrm{BW})$.

\section{Histology, Immunofluorescence and RNAscope}

All specimens for paraffin sections were fixed in $4 \%(\mathrm{w} / \mathrm{v})$ paraformaldehyde (PFA) overnight, dehydrated through an ethanol series, paraffin embedded, and sectioned $(6-7 \mu \mathrm{m})$. Primary antibodies (Supplementary Table 1) were incubated at $4{ }^{\circ} \mathrm{C}$ overnight and secondary antibodies (Alexa 488, 555, or 647, Life Technologies, Grand Island, NY, United States) were incubated at room temperature for $1 \mathrm{~h}$. The Aurkb RNAscope probe (173-1483 bp of the Mus musculus Aurkb mRNA sequence) was designed and provided by Advanced Cell Diagnostics (Hayward, CA, United States). RNAscope in situ hybridizations (Ikpa et al., 2016) were performed according to the protocol provided by manufacturer.

\section{Image Analysis and Quantification}

ImageJ software was used for quantification of GFP+ and/or BrdU+ cells on histology slides. Samples from 3-6 mice each were counted at any given time point or condition. The reported values represent the mean score. 


\section{Live Cell Imaging}

Time-lapse phase-contrast and GFP immunofluorescence images of mouse embryo fibroblasts (MEFs) were taken for $22 \mathrm{~h}$ after 4-OH tamoxifen induction (final concentration: $1 \mu \mathrm{g} / \mathrm{ml}$ ) by using the IncuCyte live-cell culture system (Essen Bioscience). The images were then analyzed and converted to movie format by using IncuCyte software.

\section{Fluorescence-Activated Cell Sorting (FACS) Analyses}

MEFs were isolated and cultured as previously described ( $\mathrm{Li}$ et al., 2011). MEFs were treated with either control vehicles or designated cell cycle inhibitors, then digested and collected as single cell suspensions. The cell suspension was washed with PBS and then fixed with intracellular fixation buffer (eBiosciences). For intracellular FACS analyses, cells were permeabilized with permeabilization buffer (eBiosciences) and then incubated with GFP antibodies (see Supplementary Table 1) for $2 \mathrm{~h}$ at room temperature, followed by incubation with secondary antibodies (Alexa fluor, Life Technologies) for $1 \mathrm{~h}$ at room temperature. Samples were run and analyzed using a BD FACS Canto II instrument and software (BD Biosciences).

\section{Quantitative Real-Time PCR (qRT-PCR)}

Heart, brain, and embryonic tissues were microdissected in cold PBS and snap frozen in liquid nitrogen. TRIzol reagent (Life Technologies, Grand Island, NY, United States) was used to extract total RNA and complementary DNA (cDNA) was generated with the Superscript III kit (Life Technologies, Grand Island, NY, United States). SYBR Green quantitative RT-PCR was performed using the StepOne Plus Real-Time PCR System (Applied Biosystems, Foster City, CA, United States). Primers for Aurkb: P1F (forward): 5'-TCGCTGTTGTTTCCCTCTCT3', P1R (reverse): 5' ${ }^{\prime}$-TTCAGGCCAGACTGAGACG-3'; P2F (forward): TCGCTGTTGTTTCCCTCTCT, P2R (reverse): TTCAGGCCAGACTGAGACG. Primers for Gapdh: Forward: 5'-TCTTGCTCAGTGTCCTTGCTGG-3', Reverse: 5' -TCCTGGTATGACAATGAATAC GGC-3'.

\section{Western Blotting}

E12.5 embryos were minced in cold lysis buffer $(50 \mathrm{mM}$ Tris$\mathrm{HCl}$ (pH 7.4), $150 \mathrm{mM} \mathrm{NaCl}, 1$ mM EDTA-Na, 1 mM EGTA, $1 \%$ Triton X-100, 0.5\% Sodium Deoxycholate and 0.1\% SDS with Protease inhibitor cocktail (Roche); $1 \mathrm{mM}$ phenylmethylsulfonyl fluoride was added before use). Protein samples were resolved on $4-12 \%$ SDS-PAGE acrylamide gel before transferring to PVDF membranes. We used primary antibodies to Aurkb (1:1000), Cre (1:1000) and GAPDH (1:5000). Primary antibodies were visualized by chemiluminescence using HRP-conjugated secondary antibodies.

\section{Statistical Analysis}

Data are presented as mean \pm SEM. Statistical significance between two groups was determined using two-tailed Student's $t$-test or chi square test. If significance is to be tested between multiple groups, an analysis of variance is performed, followed by Bonferroni post hoc test. $P<0.05$ was considered significant.

\section{RESULTS}

\section{Aurkb Is Expressed in Proliferating but Not in Quiescent Cells}

In cultured MEFs, Aurkb protein is undetectable at G1 phase, but expression becomes prominent at G2 and it is localized to the nucleus. Aurkb reaches and maintains a strong expression level throughout $\mathrm{M}$ phase. Aurkb re-localizes to the midbody at telophase (Supplementary Figure 2). These findings are consistent with previous observations (Crosio et al., 2002; Li et al., 2015). Aurkb mRNA is not detectable at G1 but is detectable by G2. Message is present through $\mathrm{M}$ phase but becomes undetectable at telophase (Figure 1). These results suggest that $A u r k b$ transcript expression is correlated with the phase of the cell cycle and is largely restricted to mitotic cells. To further test this association, we forced MEFs to arrest at G1/S phase by exposure to hydroxyurea or mimosine (Park et al., 2012) and then assessed the presence of Aurkb transcripts.

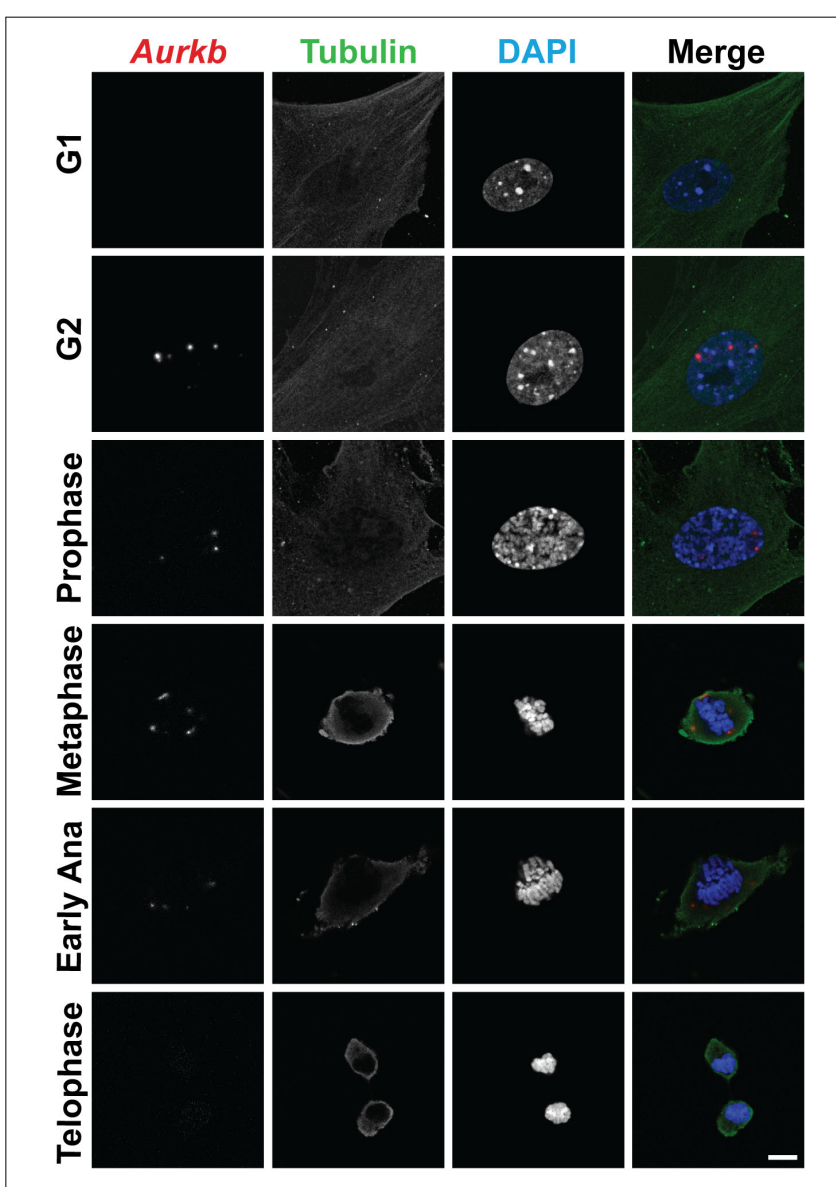

FIGURE 1 | The expression of Aurkb transcripts during the cell cycle. Double staining of RNAscope and immunofluorescence in MEFs. Bar, $10 \mu \mathrm{m}$. 
Aurkb transcriptional levels were significantly decreased in hydroxyurea- and mimosine-treated groups as compared to the control group (Figure 2A). Both brain and heart experience a proliferation transition from being highly proliferative at embryonic stage to being mostly proliferatively inert in adulthood (Brooks et al., 1998). Aurkb transcription significantly declines from being high at embryonic day (E) 14.5 to being almost undetectable in adult heart and brain (Figure 2B). Altogether, these data suggest that Aurkb transcription is coupled with cell proliferation in vitro and in vivo. Accordingly, we generated $A u r k b^{E R C r e /+}$ mice by targeting a Cre-Ert2 cassette into the start codon of the Aurkb locus (Figure 3A). As expected, $A u r k b$ mRNA was reduced to about $50 \%$ in Aurkb $b^{E R C r e /+}$ heterozygous mice as compared to their wildtype littermate controls (Figure 3C). In contrast, Aurkb protein expression was similar between $A u r k b^{E R C r e /+}$ heterozygous mice and wildtype
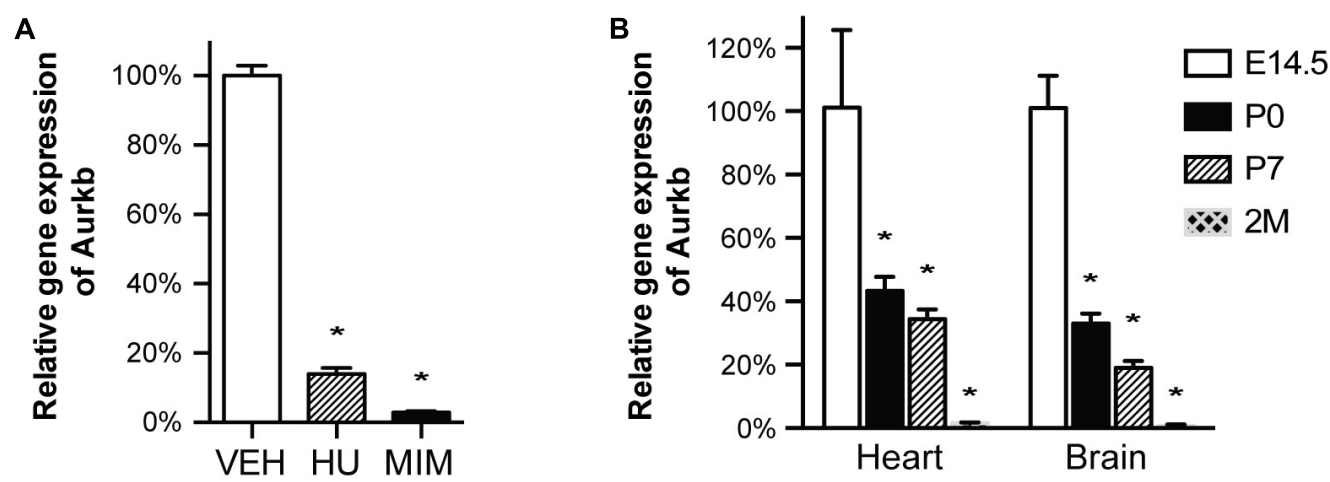

FIGURE 2 | Aurkb transcription is coupled with cell proliferation. (A) MEFs were cultured for $48 \mathrm{~h}$ with normal media (control), or treated with hydroxyurea (2 mM), or mimosine ( $1 \mathrm{mM})$. The transcriptional levels of Aurkb were quantified by qRT-PCR. Gapdh was used as a cDNA loading control. Three independent biological samples were used in each condition. ${ }^{\star} P<0.05$ when compared to the control condition analyzed by ANOVA followed by Bonferroni post hoc test; VEH, vehicle control; $\mathrm{HU}$, hydroxyurea; MIM, mimosine; (B) The relative transcriptional levels of Aurkb in heart and brain at E14.5, postnatal day 0 (P0), postnatal day 7 (P7), and 2 months (2M). Gapdh was used as a cDNA loading control. $n=3$ in each group. ${ }^{*} P<0.05$ when compared to E14.5 analyzed by ANOVA followed by Bonferroni post hoc test.

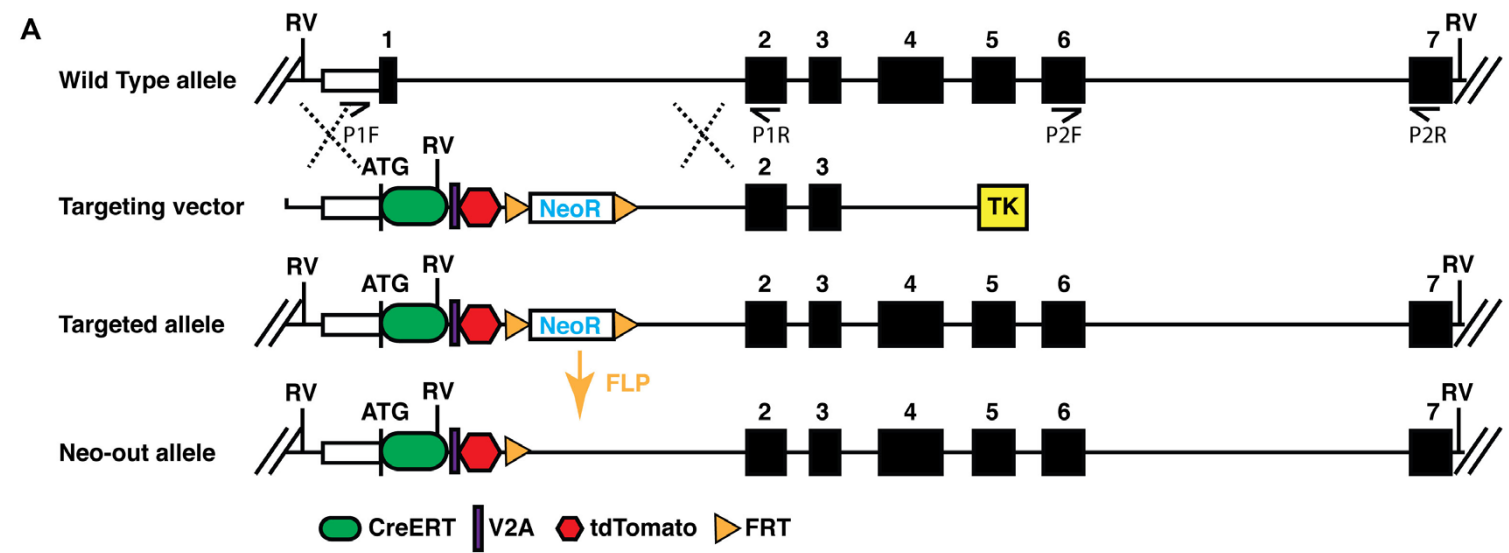

B

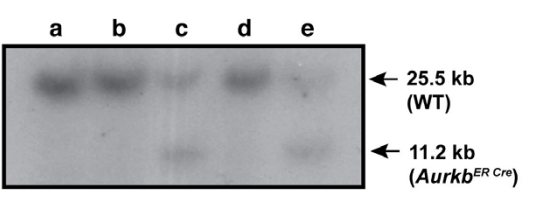

a, b, d: WT; c, e: Aurkb ${ }^{E R}$ Cre/t

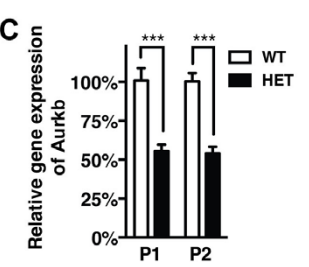

D
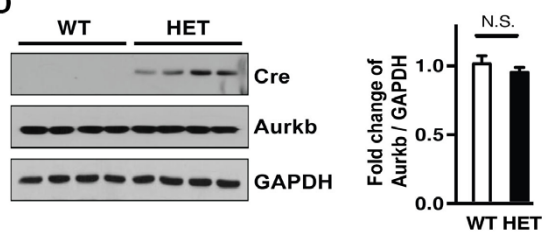

FIGURE 3 | Aurkb gene targeting. (A) Schematic of the generation of the Aurkb $b^{E R}$ Cre allele; (B) Southern blot of DNA extracted from Aurkb ${ }^{E R}$ Cre/+ MEFs. DNAs were digested by EcoRV (RV). The ${ }^{32}$ P-radiolabeled $5^{\prime}$ DNA probe used ( $500 \mathrm{bp}$ ) is located upstream of the $5^{\prime}$ arm but downstream to the $5^{\prime}$ RV enzyme site. P1F, P1R, P2F, and P2R are primer sets for detecting Aurkb transcripts; (C) Aurkb mRNA expression in wildtype (WT) and heterozygous (HET) E12.5 embryos. $n=4$ in each group. ${ }^{\star \star \star} P<0.001$ by a Student's $t$-test; (D) Aurkb protein expression in WT and HET E12.5 embryos. GAPDH was used as a protein loading control. Densitometric quantification of Aurkb was shown on the right. N.S., not significant. 
controls (Figure 3D). Aurkb $b^{E R C r e / t}$ heterozygous mice are phenotypically normal and fertile. The total knockout of Aurkb resulted in early post-implantation lethality by E9.5 (FernandezMiranda et al., 2011). Consistently, we did not recover any $A u r k b^{E R ~ C r e / E R ~ C r e ~ e m b r y o s ~ a t ~ E 10.5 ~(0 / 21) . ~}$

\section{Aurkb $^{E R}$ Cre Labels Proliferating Cells in vitro}

To characterize the labeling of $A u r k b^{E R C r e}$ in vitro and test whether it is associated with cell proliferation, we generated Aurkb ${ }^{E R C r e /+} ; \quad R 26 R^{e Y F P /+}$ MEFs and tracked Aurkb ${ }^{E R C r e}$ labeling by following the YFP reporter activities after $4-\mathrm{OH}$ tamoxifen induction. YFP signal was detectable in proliferating MEFs about $16 \mathrm{~h}$ after $4-\mathrm{OH}$ tamoxifen induction, became strong immediately prior to cell division, and maintained expression in daughter cells (Supplementary Video 1). In contrast, there was no YFP signal in non-dividing $A u r k b b^{E R C r e /+}$; $R 26 R^{e Y F P /+}$ MEFs. Note that YFP signal is well recognized by GFP antibodies. Hereafter, we use GFP antibodies to measure YFP expression when referring to $A u r k b^{E R C r e /+}$ fatemapped cells. There was negligible $R 26 R^{e Y F P /+}$ reporter activity in $\mathrm{Aurkb} b^{\mathrm{ER} C r e /+} ; \mathrm{R} 26 \mathrm{R}^{e Y F P /+} \mathrm{MEF}$ without 4-OH tamoxifen induction (Figure 4A), indicating that there is little to no leakiness of the Aurkb ${ }^{E R}$ Cre/+ allele. According to the expression profile of $A u r k b$, we expected to see $A u r k b^{E R C r e /+}$ labeling cells as they enter G2 phase. To further analyze the association between $A u r k b{ }^{E R C r e /+}$ labeling and cell proliferation, we arrested MEFs at G1/S phase by either hydroxyurea or mimosine treatment, as evidenced by the absence of BrdU incorporation (Figure 4A). $R 26 R^{e Y F P /+}$ reporter activities were significantly lower for cell cycle inhibitor-treated Aurkb $b^{E R C r e /+} ; R 26 R^{e Y F P /+}$ MEFs compared to those under normal culture conditions (Figures 4A,B). This lineage tracing result mirrors the Aurkb transcription profile when wild-type MEFs are arrested at G1/S phase (Figure 2A). These results suggest that $A u r k b^{E R ~ C r e ~ l a b e l s ~}$ proliferating but not non-dividing cells in vitro.

\section{Aurkb $^{E R}$ Cre Labels Proliferating Cells During Embryonic Development}

Next we sought to determine whether $A u r k b^{E R}$ Cre labels highly proliferative cells during embryonic development in vivo. We confirmed that Aurkb heterozygosity did not grossly affect embryonic morphogenesis or cellular growth (Supplementary Figure 3), validating the use of $A u r k b^{E R C r e /+}$ as a lineage tracing tool during embryonic development. When E8.5 Aurkb ${ }^{E R C r e /+} ; R_{2} 6^{Y Y F P /+}$ embryos were induced with tamoxifen, extensive labeling of the embryo was observed. Importantly, there was no leakiness of $A u r k b^{E R C r e}$ labeling when corn oil but not tamoxifen was administered (Supplementary Figure 4). When we labeled developing embryos with both Aurkb ${ }^{E R C r e}$ and BrdU, we found that about $85 \%$ of embryonic cells are labeled by both systems (Figure 5). Further, we performed double immunofluorescence staining of GFP and PCNA on these embryos. We found that nearly $93 \%$ of embryonic cells are double positive (Supplementary Figure 5). Altogether, these data indicate that $A u r k b^{E R C r e}$ is a sensitive
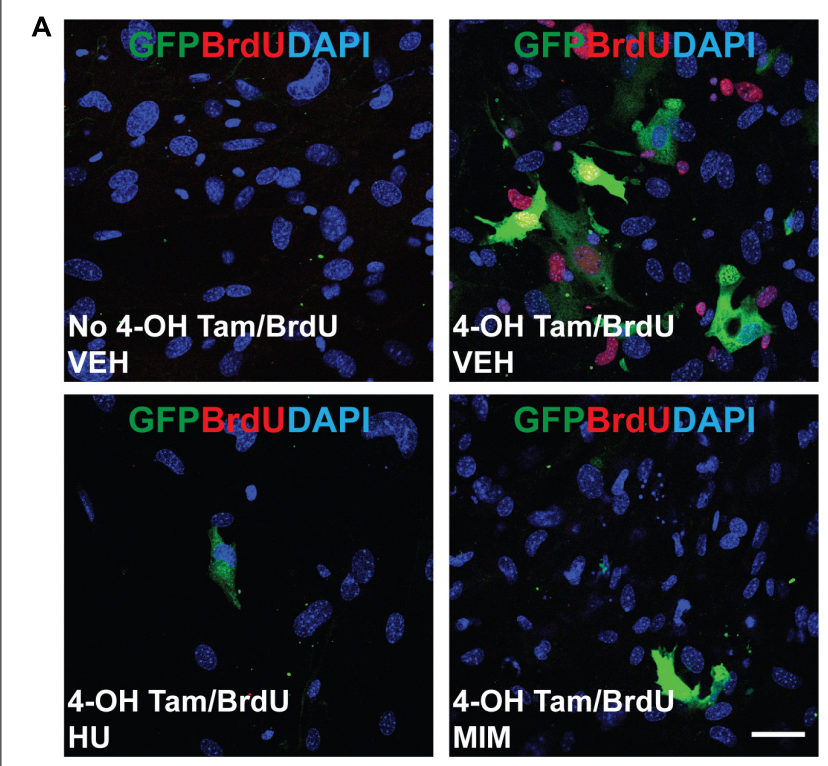

B

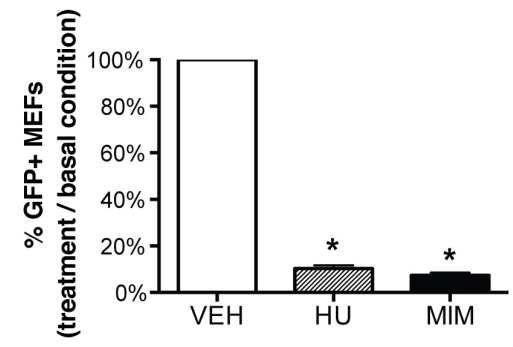

FIGURE 4 | AurkbER Cre/+ labeling of MEFs. Aurkb ${ }^{E R ~ C r e /+; ~ R 26 R ~} R^{e Y F P /+}$ MEFs were cultured in 10\% FBS standard medium. MEFs were treated with cell cycle blockers for a total of $48 \mathrm{~h}$. $24 \mathrm{~h}$ after the treatment, BrdU $(10 \mu \mathrm{M})$ and $4-\mathrm{OH}$ tamoxifen $(1 \mu \mathrm{M})$ were added in the medium and maintained for $24 \mathrm{~h}$ before cell harvest. YFP immunosignal was detected by GFP antibody. (A) Representative micrographs of BrdU and GFP immunofluorescence staining of Aurkb $b^{E R ~ C r e /+} ; R 26 R^{e Y F P /+}$ MEFs under various culture conditions. Scale bar, $50 \mu \mathrm{m}$; (B) The AurkbER Cre/+ labeling percentage was quantified by flow cytometric analysis. The percentage was calculated as the number of GFP + cells under each treatment condition as divided by the number of GFP + cells in the control condition. VEH, vehicle control; HU, hydroxyurea (2 mM); MIM, mimosine ( $1 \mathrm{mM}) . n=3$ for each condition, ${ }^{\star} P<0.05$ when compared to the control group by ANOVA followed by Bonferroni post hoc test

and reliable system for lineage tracking of proliferating embryonic cells.

\section{Tamoxifen Activation of Aurkb ${ }^{E R}$ Cre Labels Proliferating Adult Stem/Progenitor Cells but Not Post-mitotic Cells in vivo}

Next, we assessed $A u r k b^{E R C r e}$ labeling in adult regenerative tissues. Two-month-old $A u r k b^{E R C r e /+} ; R_{2} 6^{e Y F P /+}$ mice were given a single dose of tamoxifen (100 mg/kg BW) and we followed the labeling pattern of the YFP reporter over time in the intestine. The labeling displayed a dynamic expansion 


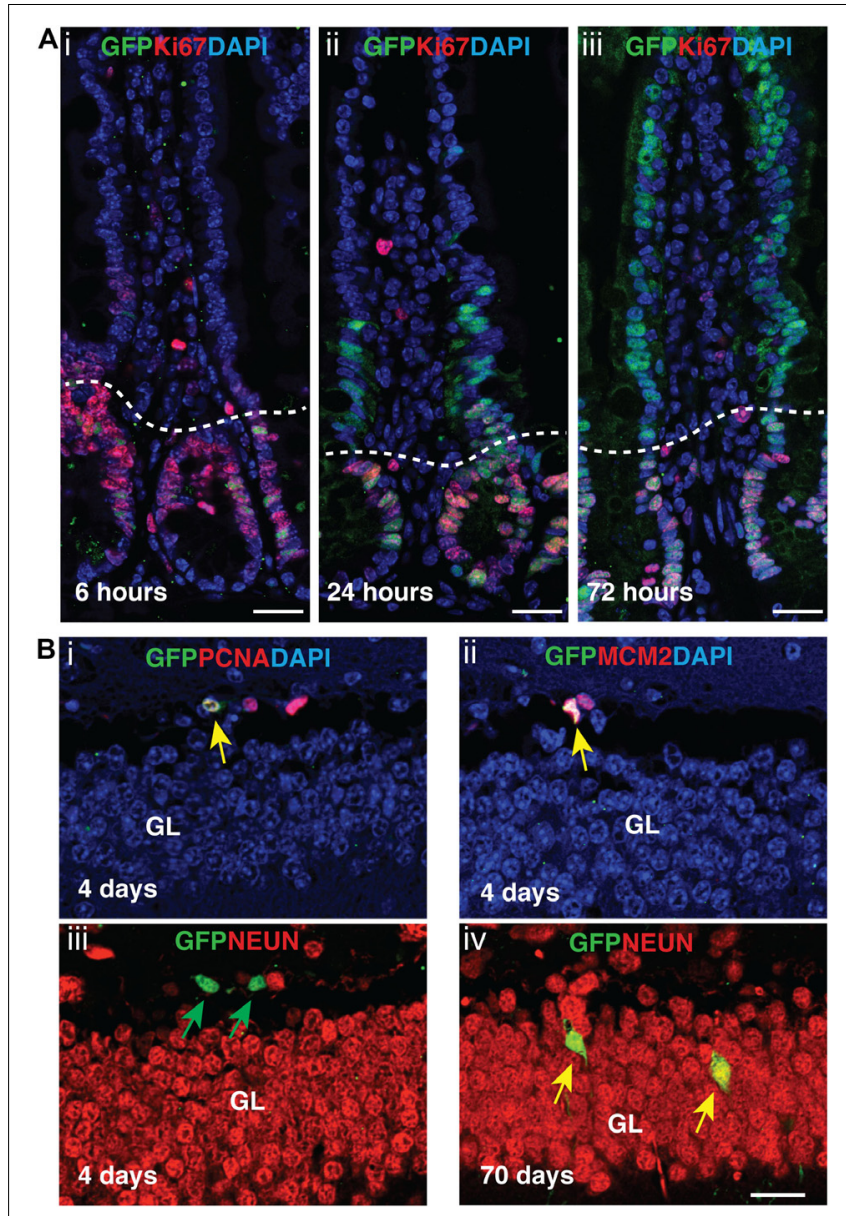

FIGURE 6 | Tamoxifen activation of Aurkb $b^{E R}$ Cre/+ labels proliferating stem cells/progenitor cells in adult intestines and brain. (A) Aurkb $b^{E R ~ C r e /+}$ labeling in adult intestines. A single dose of tamoxifen (100 mg/kg BW, i-iii) was intraperitoneally given to Aurkb $b^{E R ~ C r e /+} ; R 26 R^{\text {YYFP } /+}$ mice at the age of 2 months. Dotted lines delineate the crypts below from their associated villus above. Bars, $25 \mu \mathrm{m}$; (B) AurkbER Cre/+ labeling in adult brain. Tamoxifen (100 mg/kg BW) was intraperitoneally given daily to Aurkb ER Cre/+; $R 26 R^{\text {YYFP } /+}$ mice for four consecutive days at the age of 2 months. Green arrows point to GFP+ only cells; yellow arrows point to GFP+; PCNA+ (i), GFP+; MCM2+ (ii) or GFP+; NeuN+ (iv) cells. GL, granular layer. Bars, 20 um.

cells. However, increased focus on adult tissue regeneration calls for new tools to enable the detection of tissues and cell lineages derived from proliferating adult progenitor cells under various conditions. The MADM mouse can be used for tracking populations derived from proliferative progenitors. However, its application is limited due to its low detection sensitivity (Zong, 2014). A Ki67 IRESCreER mouse tracks proliferating cells based on Ki67 expression (Basak et al., 2018). However, Ki67 is transcribed broadly throughout the cell cycle, a feature that appears a critical factor in its reliability as a proliferation marker (Miller et al., 2018). In this report, we describe a new genetic mouse tool, $A u r k b^{E R ~ C r e /+}$, which can label cells based on Aurkb transcription. We found that $A u r k b^{E R C r e}$ labels proliferating cells in vitro and in vivo. In developing embryos, Aurkb $b^{E R}$ Cre labeling overlaps well with cell proliferation markers such as
BrdU and PCNA but it does not label quiescent cells. We notice that the overlap between $A u r k b^{E R}$ Cre labeling and BrdU or PCNA seems quite high during development, even though $A u r k b^{E R C r e}$ labeling was induced by a short pulse of tamoxifen. This is primarily due to the high proliferative characteristics of developing embryos: both earlier proliferating embryonic cells and their later derivatives are all mitotic.

Since $A u r k b^{E R}$ Cre labeling is based on Aurkb transcription, which turns on at G2 phase, Aurkb ${ }^{E R}$ Cre labeling should not be interpreted as an absolute cell division or cytokinetic marker. For instance, certain cells such as bi- or multinucleated adult cardiomyocytes and hepatocytes can undergo karyokinesis without cytokinesis under physiological or pathological conditions (Gentric et al., 2012; Derks and Bergmann, 2020). These cells may be labeled by $A u r k b^{E R C r e}$ in the absence of cell division. In these specific instances, Aurkb protein expression and other cytokinetic events should be analyzed instead.

Since $A u r k b^{E R C r e /+}$ is built by a knock-in strategy, the Aurkb transcript is disrupted in the targeted allele. In contrast, Aurkb protein expression level in $A u r k b^{E R C r e /+}$ heterozygous mice was quite similar to their wildtype controls (Figure 3D). This is consistent with other reports that heterozygous knockout mice can express similar or more than half of the proteins relative to wildtype control mice in the target genes (Gineste et al., 2013; Zhou et al., 2015; Arkhipov et al., 2019). It is reported that an Aurkb knockout mouse in which exons 2-6 were excised is embryonic lethal, older Aurkb heterozygous mice approximately 12-24 months of age show decreased survival due to susceptibility for tumorigenesis, and a fraction of Aurkb heterozygous males suffer from oligospermia by 12 months of age (Fernandez-Miranda et al., 2011). In our current study, we used much younger mice less than 3-months of age. We did not observe reproductive defects or spontaneous cancer development in young $\mathrm{Aurkb} b^{E R C r e /+}$ mice. Nonetheless, it is possible that $A u r k b^{E R C r e /+}$ mice may develop aforementioned pathologies over time, due to genome or chromosomal instability, even though we did not observe such problems in our colony. On the other hand, by intercrossing Aurkb ${ }^{E R ~ C r e /+}$ heterozygous mice, the progeny with genotype of $A u r k b^{E R}$ Cre/ER Cre can be used to study the phenotype of Aurkb depletion or requirement of Aurkb expression in cell division and cytokinesis.

The Aurkb $b^{E R C r e /+}$ mouse is an important new tool for lineage tracing proliferating cells during embryonic development and adult tissue regeneration. For instance, when crossed to multicolor reporters such as the confetti mouse strain (Snippert et al., 2010), the Aurkb ${ }^{E R C r e /+}$ mouse can be used to study the clonogenicity of neurons or other cell types in the developing embryos or tissue regeneration (e.g., whether cells are derived from multiple progenitor cells or from rare dominant clones). Similarly, the $A u r k b^{E R C r e /+}$ mouse can be used to track tissue regeneration such as occurring from those rare stem cells whose derivatives forming over long periods of time will be more readily detected with this tool. The $A u r k b^{E R C r e /+}$ mouse allows the ability to track how tissues with low rates of proliferation such as brain, lung and heart are regenerated over time in various physiological or pathological conditions. We anticipate the coupling of Aurkb knockout with inducible Cre-mediated 
capability will be a powerful reagent for the investigation of cell proliferation in the context of Aurkb expression.

\section{DATA AVAILABILITY STATEMENT}

All datasets generated for this study are included in the article/Supplementary Material.

\section{ETHICS STATEMENT}

The animal studies were reviewed and approved by the University of Pennsylvania Institutional Animal Care and Use Committee and the University of Maryland Baltimore Institutional Animal Care and Use Committee.

\section{AUTHOR CONTRIBUTIONS}

JE and DL conceived and designed the study. JJ, KE, and DL analyzed the experiments and wrote the original draft. JJ, KE, JE, and DL edited and finalized the manuscript. JJ, KE, FL, LL, GS, and DL performed the experiments. All authors reviewed the results and approved the final version of the manuscript.

\section{REFERENCES}

Alvarez, R. Jr., Wang, B. J., Quijada, P. J., Avitabile, D., and Ho, T. (2019). Cardiomyocyte cell cycle dynamics and proliferation revealed through cardiacspecific transgenesis of fluorescent ubiquitinated cell cycle indicator (FUCCI). J. Mol. Cell Cardiol. 127, 154-164. doi: 10.1016/j.yjmcc.2018.12.007

Arkhipov, S. N., Potter, D. L., Geurts, A. M., and Pavlov, T. S. (2019). Knockout of P2rx7 purinergic receptor attenuates cyst growth in a rat model of ARPKD. Am. J. Physiol. Renal Physiol. 317, F1649-F1655. doi: 10.1152/ajprenal.00395.2019

Barker, N. (2014). Adult intestinal stem cells: critical drivers of epithelial homeostasis and regeneration. Nat. Rev. Mol. Cell Biol. 15, 19-33. doi: 10.1038/ nrm3721

Basak, O., Krieger, T. G., Muraro, M. J., Wiebrands, K., Stange, D. E., FriasAldeguer, J., et al. (2018). Troy+ brain stem cells cycle through quiescence and regulate their number by sensing niche occupancy. Proc. Natl. Acad. Sci. U.S.A. 115, E610-E619. doi: 10.1073/pnas.1715911114

Bond, A. M., Ming, G. L., and Song, H. (2015). Adult mammalian neural stem cells and neurogenesis: five decades later. Cell Stem Cell 17, 385-395. doi: 10.1016/j.stem.2015.09.003

Brooks, G., Poolman, R. A., and Li, J. M. (1998). Arresting developments in the cardiac myocyte cell cycle: role of cyclin-dependent kinase inhibitors. Cardiovasc. Res. 39, 301-311. doi: 10.1016/s0008-6363(98)00125-4

Crosio, C., Fimia, G. M., Loury, R., Kimura, M., Okano, Y., Zhou, H., et al. (2002). Mitotic phosphorylation of histone H3: spatio-temporal regulation by mammalian Aurora kinases. Mol. Cell Biol. 22, 874-885. doi: 10.1128/mcb.22.3. 874-885.2002

Derks, W., and Bergmann, O. (2020). Polyploidy in cardiomyocytes: roadblock to heart regeneration? Circ. Res. 126, 552-565. doi: 10.1161/CIRCRESAHA.119. 315408

Fernandez-Miranda, G., Trakala, M., Martin, J., Escobar, B., Gonzalez, A., Ghyselinck, N. B., et al. (2011). Genetic disruption of aurora B uncovers an essential role for aurora $\mathrm{C}$ during early mammalian development. Development 138, 2661-2672. doi: 10.1242/dev.066381

Gentric, G., Celton-Morizur, S., and Desdouets, C. (2012). Polyploidy and liver proliferation. Clin. Res. Hepatol. Gastroenterol. 36, 29-34. doi: 10.1016/j.clinre. 2011.05.011

\section{FUNDING}

This work was supported by the American Heart Association Scientist Development Grant (17SDG33650102) and department seed fund to DL; the Cotswold Foundation, the WW Smith Endowed Chair, and the National Institutes of Health (NIH) grant R35 HL140018 to JE.

\section{ACKNOWLEDGMENTS}

We thank the financial support from Department of Surgery, University of Maryland School of Medicine. We thank the Penn Cardiovascular Institute Histology Core for technical assistance, and the Penn CDB Microscopy Core for confocal imaging. We also thank Dr. Weinian Shou, Indiana University School of Medicine, for advice on the manuscript.

\section{SUPPLEMENTARY MATERIAL}

The Supplementary Material for this article can be found online at: https://www.frontiersin.org/articles/10.3389/fcell.2020.00388/ full\#supplementary-material

Gineste, C., De Winter, J. M., Kohl, C., Witt, C. C., Giannesini, B., Brohm, K., et al. (2013). In vivo and in vitro investigations of heterozygous nebulin knock-out mice disclose a mild skeletal muscle phenotype. Neuromuscul. Disord. 23, 357-369. doi: 10.1016/j.nmd.2012.12.011

Helfrich, B. A., Kim, J., Gao, D., Chan, D. C., Zhang, Z., Tan, A. C., et al. (2016). Barasertib (AZD1152), a small molecule aurora b inhibitor, inhibits the growth of SCLC cell lines in vitro and in vivo. Mol. Cancer Ther. 15, 2314-2322. doi: 10.1158/1535-7163.MCT-16-0298

Ikpa, P. T., Sleddens, H. F., Steinbrecher, K. A., Peppelenbosch, M. P., de Jonge, H. R., Smits, R., et al. (2016). Guanylin and uroguanylin are produced by mouse intestinal epithelial cells of columnar and secretory lineage. Histochem. Cell. Biol. 146, 445-455. doi: 10.1007/s00418-0161453-4

Kretzschmar, K., Post, Y., Bannier-Helaouet, M., Mattiotti, A., Drost, J., Basak, O., et al. (2018). Profiling proliferative cells and their progeny in damaged murine hearts. Proc. Natl. Acad. Sci. U.S.A. 115, E12245-E12254. doi: 10.1073/pnas. 1805829115

Li, D., Hallett, M. A., Zhu, W., Rubart, M., Liu, Y., Yang, Z., et al. (2011). Dishevelled-associated activator of morphogenesis 1 (Daam1) is required for heart morphogenesis. Development 138, 303-315. doi: 10.1242/dev.055566

Li, S., Deng, Z., Fu, J., Xu, C., Xin, G., Wu, Z., et al. (2015). Spatial Compartmentalization Specializes the Function of Aurora A and Aurora B. J. Biol. Chem. 290, 17546-17558. doi: 10.1074/jbc.M115.652453

Liu, D., and Lampson, M. A. (2009). Regulation of kinetochore-microtubule attachments by Aurora B kinase. Biochem. Soc. Trans. 37(Pt 5), 976-980. doi: 10.1042/BST0370976

Miller, I., Min, M., Yang, C., Tian, C., Gookin, S., Carter, D., et al. (2018). Ki67 is a graded rather than a binary marker of proliferation versus quiescence. Cell Rep. 24, 1105-1112. doi: 10.1016/j.celrep.2018.06.110

Mitchison, T. J., and Salmon, E. D. (2001). Mitosis: a history of division. Nat. Cell Biol. 3, E17-E21. doi: 10.1038/35050656

Nakada, Y., Canseco, D. C., Thet, S., Abdisalaam, S., Asaithamby, A., Santos, C. X., et al. (2017). Hypoxia induces heart regeneration in adult mice. Nature 541, 222-227. doi: 10.1038/nature20173

Nasmyth, K. (2001). A prize for proliferation. Cell 107, 689-701. doi: 10.1016/ s0092-8674(01)00604-3 
Pan, Q., Nicholson, A. M., Barr, H., Harrison, L. A., Wilson, G. D., Burkert, J., et al. (2013). Identification of lineage-uncommitted, long-lived, label-retaining cells in healthy human esophagus and stomach, and in metaplastic esophagus. Gastroenterology 144, 761-770. doi: 10.1053/j.gastro.2012.12.022

Park, S. Y., Im, J. S., Park, S. R., Kim, S. E., Wang, H. J., and Lee, J. K. (2012). Mimosine arrests the cell cycle prior to the onset of DNA replication by preventing the binding of human Ctf4/And-1 to chromatin via Hif-1alpha activation in HeLa cells. Cell Cycle 11, 761-766. doi: 10.4161/cc.11.4.19209

Rodriguez, C. I., Buchholz, F., Galloway, J., Sequerra, R., Kasper, J., Ayala, R., et al. (2000). High-efficiency deleter mice show that FLPe is an alternative to Cre-loxP. Nat. Genet. 25, 139-140. doi: 10.1038/75973

Snippert, H. J., van der Flier, L. G., Sato, T., van Es, J. H., van den Born, M., KroonVeenboer, C., et al. (2010). Intestinal crypt homeostasis results from neutral competition between symmetrically dividing Lgr5 stem cells. Cell 143, 134-144. doi: 10.1016/j.cell.2010.09.016

Tang, A., Gao, K., Chu, L., Zhang, R., Yang, J., and Zheng, J. (2017). Aurora kinases: novel therapy targets in cancers. Oncotarget 8, 23937-23954. doi: 10.18632/ oncotarget.14893

Tian, Y., Liu, Y., Wang, T., Zhou, N., Kong, J., Chen, L., et al. (2015). A microRNA-Hippo pathway that promotes cardiomyocyte proliferation and cardiac regeneration in mice. Sci. Transl. Med. 7:279ra238. doi: 10.1126/ scitranslmed.3010841

Tischer, J., and Gergely, F. (2019). Anti-mitotic therapies in cancer. J. Cell Biol. 218, 10-11. doi: 10.1083/jcb.201808077

Vader, G., and Lens, S. M. (2008). The Aurora kinase family in cell division and cancer. Biochim. Biophys. Acta 1786, 60-72. doi: 10.1016/j.bbcan.2008.07.003

van der Waal, M. S., Hengeveld, R. C., van der Horst, A., and Lens, S. M. (2012). Cell division control by the chromosomal passenger complex. Exp. Cell Res. 318, 1407-1420. doi: 10.1016/j.yexcr.2012.03.015
Yu, J. J., Zhou, L. D., Zhao, T. T., Bai, W., Zhou, J., and Zhang, W. (2015). Knockdown of aurora-b inhibits the growth of non-small cell lung cancer A549 cells. Oncol. Lett. 10, 1642-1648. doi: 10.3892/ol.2015. 3467

Yu, K. W., Zhong, N., Xiao, Y., and She, Z. Y. (2019). Mechanisms of kinesin-7 CENP-E in kinetochore-microtubule capture and chromosome alignment during cell division. Biol. Cell 111, 143-160. doi: 10.1111/boc.2018 00082

Zhou, C., Ding, L., Deel, M. E., Ferrick, E. A., Emeson, R. B., and Gallagher, M. J. (2015). Altered intrathalamic GABAA neurotransmission in a mouse model of a human genetic absence epilepsy syndrome. Neurobiol. Dis. 73, 407-417. doi: 10.1016/j.nbd.2014.10.021

Zong, H. (2014). Generation and applications of MADM-based mouse genetic mosaic system. Methods Mol. Biol. 1194, 187-201. doi: 10.1007/978-1-49391215-5_10

Zong, H., Espinosa, J. S., Su, H. H., Muzumdar, M. D., and Luo, L. (2005). Mosaic analysis with double markers in mice. Cell 121, 479-492. doi: 10.1016/j.cell. 2005.02 .012

Conflict of Interest: The authors declare that the research was conducted in the absence of any commercial or financial relationships that could be construed as a potential conflict of interest.

Copyright $\odot 2020$ Jang, Engleka, Liu, Li, Song, Epstein and Li. This is an open-access article distributed under the terms of the Creative Commons Attribution License (CC BY). The use, distribution or reproduction in other forums is permitted, provided the original author(s) and the copyright owner(s) are credited and that the original publication in this journal is cited, in accordance with accepted academic practice. No use, distribution or reproduction is permitted which does not comply with these terms. 